\title{
Validation of non-invasive tools in predicting HBV-related acute-on-chronic liver failure
}

\author{
Ting $\mathrm{Wu}^{1} \cdot$ Tao $^{\mathrm{Chen}}{ }^{1} \cdot$ Qin Ning $^{1}$ (1) \\ Received: 20 January 2021 / Accepted: 27 March 2021 / Published online: 17 June 2021 \\ (c) Asian Pacific Association for the Study of the Liver 2021
}

\section{Introduction}

Acute-on-chronic liver failure (ACLF) is an acute hepatic insult in a patient with chronic liver disease characterized by its rapid progression and high incidence of short- and medium-term mortality of 50-90\%. While in Asia-Pacific region, chronic hepatitis $\mathrm{B}$ is the major etiological risk factor for chronic liver disease, and hepatitis B virus (HBV) reactivation remains the leading cause of acute hepatic insult. Therefore, the accurate prediction of HBV-related ACLF is important for the subsequent management for improved outcome in the Asian Pacific countries. The progression to ACLF depends upon the severity of liver inflammation and underlying fibrosis which represents the severity of acute hepatic insult and degree of chronic liver injury, respectively. Nevertheless, liver biopsy is not usually performed in the setting of acute exacerbation because of the risk of hemorrhage. Non-invasive tools thus have been investigated to indicate the risk of development to HBV-related ACLF and help prevent the deterioration.

\section{Non-invasive tools to predict HBV-related ACLF}

It has been shown pre-existing liver cirrhosis, patient age, alanine aminotransferase (ALT), aspartate aminotransferase (AST), total bilirubin (TBIL), prothrombin activity (PTA) or international normalized ratio (INR) of prothrombin time

Qin Ning

qning@vip.sina.com

Ting Wu

95359302@qq.com

Tao Chen

chentao_tjh@vip.sina.com

1 Department and Institute of Infectious Disease, Tongji Hospital, Tongji Medical College, Huazhong University of Science and Technology, 1095, Jiefang Avenue, Wuhan 430030, Hubei Province, China
(PT), albumin (ALB), ammonia, alpha fetoprotein (AFP) and HBV DNA were revealed to be related to the development to HBV-related ACLF [1-3]. Nevertheless, most of these clinical biochemical indicators were not specifically designed to predict HBV-related ACLF.

As individual indicator has limited predictive accuracy, scoring systems assembling important parameters are expected to be optimal. Model for End-Stage Liver Disease (MELD) scores containing conventional parameters (serum creatinine, INR, TBIL) was aimed to assess the liver disease severity and predict the prognosis of ACLF. The Chronic Liver Failure Consortium Organ Failure score (CLIF-C OFs) was established based on the sequential organ failure assessment (SOFA), and it showed comparable capacity to the CLIF SOFA and was superior to the MELDs and MELDNas in ACLF patients in intensive care units. The CLIF-C OFs and two other independent predictors of mortality (age and white blood cell count) were combined to develop a specific prognostic score for ACLF, the CLIF Consortium ACLF score (CLIF-C ACLFs) [4]. The AARC-ACLFs include the TBIL, hepatic encephalopathy grade, PT-INR, lactate and creatinine as parameters that showed adequate prognostic value in the overall AARC database [4]. However, those three predictive models were developed from cohorts with a small portion of HBV-related ACLF patients.

Recently, The Tongji Prognostic Predictive Model (TPPM) uses the TBIL, INR, HBV DNA and number(s) of complications as parameters as TPPM score $p=1 /\left(1+\mathrm{e}^{-\operatorname{logit}(p)}\right), \operatorname{logit}(p)=0.0031 / \mathrm{TBIL}$ $(\mu \mathrm{mol} / \mathrm{L})]+0.9519 \times \mathrm{INR}+2.2580 \times$ constant for complications( 0 if without or with 1 complication; 1 with 2 or more complications $)+0.114 \times[\lg \mathrm{HBV}$ DNA(copies $/ \mathrm{mL})]$ $-5.012[5,6]$, which showed good prognostic capacity in patients with HBV-related ACLF. The COSSH-ACLF was created based on the TBIL, INR, age and HBV-SOFA, and it was modified according to the CLIF-SOFA criteria and verified in a Chinese HBV-related ACLF cohort [7]. Compared with predictive models mentioned above, the TPPMs 
possess highest predictive ability for mortality in HBVrelated ACLF patients in a 23-center international validation cohort with 985 cases [8].

Among these scoring systems, only MELDs has been evaluated to predict the progression to HBV-related ACLF. Although high MELDs was associated with progression to ACLF, the predictive value of MELDs was not satisfying with the AUROC ranged from 0.601 to $0.820[1-3,9]$. There are currently a few specifically designed predictive models for the progression of HBV-related ACLF superior to MELDs. Gao et al. [3] developed integer risk scores of HBV-related ACLF predictors estimated from the following four parameters: age $\geq 40$ years (risk score $=1$ ), TBIL $\geq$ $171 \mu \mathrm{mol} / \mathrm{L}$ (risk score $=1$ ), PTA $40 \%-60 \%$ (risk score $=3$ if PTA $<50 \%$, otherwise $=2$ ), and HBV DNA $>10^{7}$ copies $/ \mathrm{mL}$ (risk score $=2$ ). The sum risk score ranged from 0 to 7, and 4-7 identified patients with higher risk of ACLF (26.0\%-68.8\%). Another two prediction models established by Li et al. [1] (containing age, PTA, TBIL, bilirubin, Na and HBV DNA) and Yuan et al. [2] (containing age and HBV DNA) could predict HBV-related ACLF more effectively than MELDs.

Currently, non-invasive fibrosis models have been made use of to predict HBV-related ACLF in patients with acute exacerbation and severe acute exacerbation [9]. The results demonstrated that different thresholds of liver fibrosis are required to determine the development to HBV-related ACLF in patients with different degrees of liver injury. Cirrhosis is essential to induce ACLF in case of acute exacerbation; meanwhile, lower grade of liver fibrosis could also lead to ACLF in the setting of severe acute exacerbation.

\section{Are non-invasive fibrosis models competent in predicting $\mathrm{HBV}$-related ACLF?}

In the study published in this issue of Hepatology International, only Lok index was an independent risk factor associated with progression to ACLF among the four candidate non-invasive fibrosis models: AST-to-platelet ratio index (APRI), Fibrosis-4 (FIB-4), Forns index and Lok index [9]. Thus, the validation of non-invasive fibrosis serum panels in predicting ACLF is questionable.

It is known that the diagnostic accuracy of non-invasive fibrosis serum panels is influenced in the context of liver inflammation and intrahepatic cholestasis which is present in the prelude of ACLF. This may be attributed to the inclusion of aminotransferases and/or cholestatic indicator in these serum panels. For example: APRI $=\mathrm{AST} \times 100 /$ platelet $(\mathrm{PLT}) ;$ FIB $-4=($ age $\times$ AST $) /($ platelet $\times \sqrt{ }$ ALT $)$; Forns index $=7.811-3.131 \times \ln (\mathrm{PLT})+0.781 \times \ln ($ glutamyl transpeptidase $)+3.467 \times \ln ($ age $)-0.014 \times$ total cholesterol; Lok index $=\exp (\log$ odds $) /[1+\exp (\log$ odds $)], \log$ odds $=-$ $5.56-(0.0089 \times$ PLT $)+(1.26 \times$ AST/ALT $)+(5.27 \times$ INR $)$.
As compensation, when the candidate panels are utilized, we should re-evaluate the diagnostic accuracy and re-establish the cut-off values initially in patients with severe liver inflammation to ensure the feasibility. Additionally, the association of Lok index to ACLF may not rely on the fibrosis only. This association may be attributed to the inclusion of INR as a component in Lok index, which defines liver failure and is not included in the other three panels. In some sense, the Lok index somehow is similar to MELD score rather than a fibrosis indicator. Thus, the further question is what kind of noninvasive fibrosis assessment tool is the better indicator.

Liver stiffness measurement (LSM) is an alternative tool to quantify liver fibrosis besides above biomarker panels. However, the accuracy to assess the liver fibrosis has been reported to be influenced in the presence of inflammation. Patients with the same fibrosis staging but higher ALT levels tend to have higher LSM. Consequently, different LSM cutoff values should be established for normal and elevated ALT levels. LSM by transient elastography (Fibroscan) is therefore hopeful to assess fibrosis and predict HBV-related ACLF in the patients with severe hepatitis flare. Unfortunately, there was no LSM cutoff values derived for ALT levels more than five times upper limit of normal (ULN) because biopsy was unavailable from these patients.

It may not be necessary to set the LSM cutoff values in different ALT levels, since LSM reflects liver fibrosis and inflammation which both dictate the progression to ACLF. Thus, LSM is a reliable tool to assess fibrosis in the absence of inflammation, while a potential parameter to predict ACLF in case of severe liver inflammation. Recently, the study by Jin et al. [10] used LSM by two-dimensional shear wave elastography (2D SWE) to determine prognosis of HBV-related ACLF. Different from transient elastography (Fibroscan), 2D SWE can be applied in patients with ascites which is prevalent during severe hepatitis or cirrhosis. As a result, 2D SWE is more suitable for obtaining LSMs in ACLF or its prelude. In this study, 2D SWE exhibited an efficacy comparable with that of MELDs to predict HBVrelated ACLF. Moreover, 2D SWE was combined with MELD score to create the new MELD-SWE score: MELD$\mathrm{SWE}=1.3 \times \mathrm{MELD}+0.3 \times 2 \mathrm{D}$ SWE $(\mathrm{kPa})$. The MELDSWE score showed a significantly higher prognostic value than MELDs and 2D SWE alone. This study implies the feasibility of LSM, as well as its combination with other serum models to predict HBV-related ACLF.

\section{Conclusions and future directions}

Several non-invasive indicators and models to predict HBV-related ACLF in commonly clinical use have been reviewed here (Fig. 1). However, most of the predictors 
Fig. 1 Overview of non-invasive predictors of progression to HBV-related acute-on-chronic liver failure

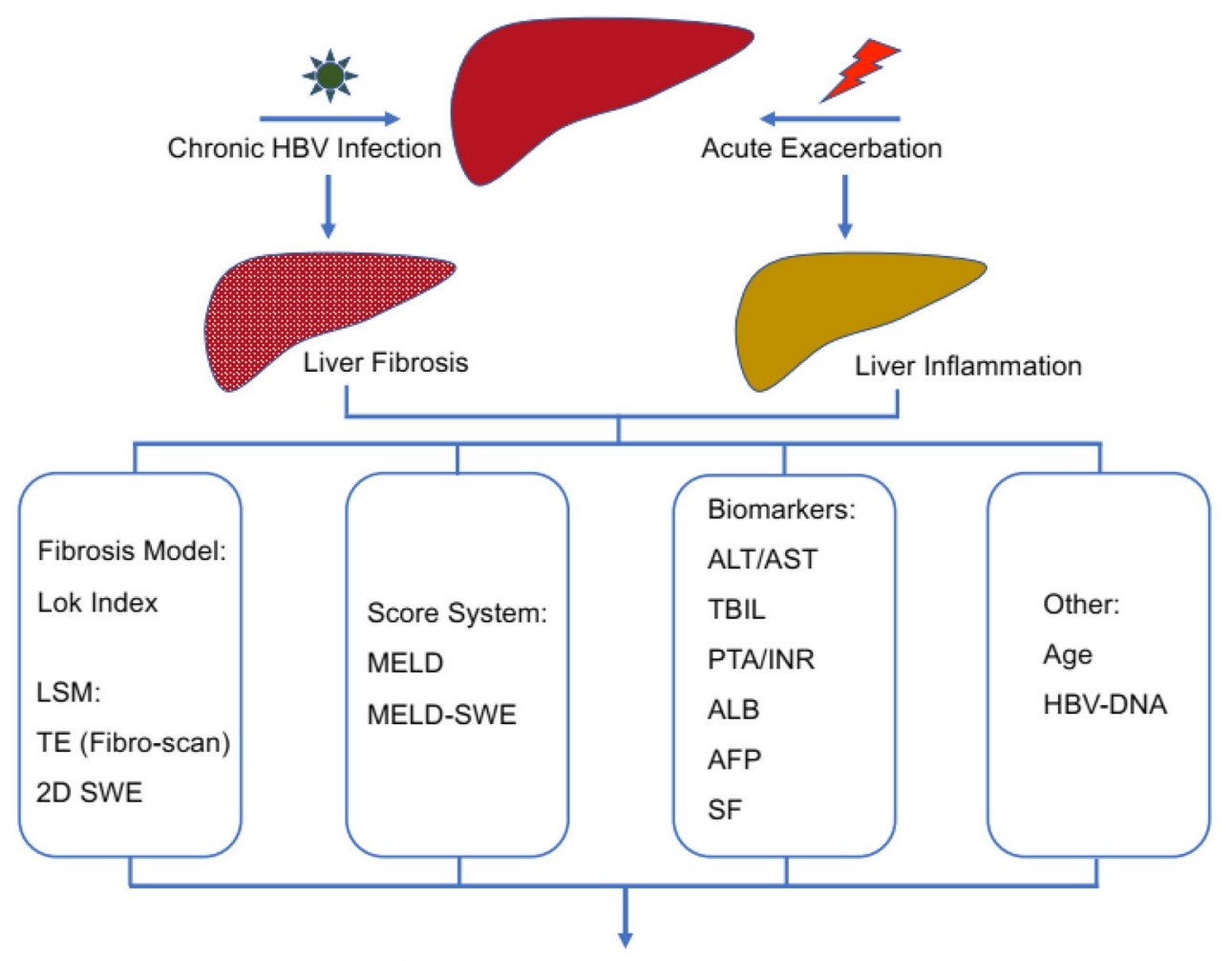

HBV related acute-on-chronic liver failure were not designed specifically to predict the progression for HBV-related ACLF. In the searching of the more specific and effective models, we should give more concern on the non-invasive fibrosis tools. Some serum panels, except Lok index, have been confirmed associated to the progression to HBV-related ACLF. But the exact relationship of Lok index and ACLF is debatable considering the meaning of INR. LSM is an alternative non-invasive fibrosis assessing tool. As LSM is related to both fibrosis and inflammation, we should determine the LSM thresholds according to different severities of hepatic injury, or directly use LSM as an ACLF indicator. On the other hand, the corporation of LSM and other ACLF predictive models need further evaluation.

\section{References}

1. Li C, Zhu B, Lv S, You S, Xin S. Prediction model of the progression of patients with acute deterioration of hepatitis $B$ virus-related chronic liver disease to acute-on-chronic liver failure, (in eng). Medicine (Baltimore) 2018;97(34):e11915. https://doi.org/10.1097/MD.0000000000011915

2. Yuan L, Zeng BM, Liu LL, Ren Y, Yang YQ, Chu J, et al. Risk factors for progression to acute-on-chronic liver failure during severe acute exacerbation of chronic hepatitis B virus infection. World J Gastroenterol 2019;25(19):2327-2337. https://doi.org/ 10.3748/wjg.v25.i19.2327

3. Gao FY, Liu Y, Li XS, Ye XQ, Sun L, Geng MF, et al. Score model for predicting acute-on-chronic liver failure risk in chronic hepatitis B. World J Gastroenterol 2015;21(27):83738381. https://doi.org/10.3748/wjg.v21.i27.8373

4. Sarin SK, Choudhury A, Sharma MK, Maiwall R, Al Mahtab M, Rahman S, et al. Acute-on-chronic liver failure: consensus recommendations of the Asian Pacific association for the study of the liver (APASL): an update. Hepatol Int 2019;13(4):353-390. https://doi.org/10.1007/s12072-019-09946-3

5. Wang J, Ma K, Han M, Guo W, Huang J, Yang D, et al. Nucleoside analogs prevent disease progression in HBVrelated acute-on-chronic liver failure: validation of the TPPM model. Hepatol Int 2014;8(1):64-71. https://doi.org/10.1007/ s12072-013-9485-5

6. Ma K, Guo W, Han M, Chen G, Chen T, Wu Z, et al. Entecavir treatment prevents disease progression in hepatitis $\mathrm{B}$ virusrelated acute-on-chronic liver failure: establishment of a novel logistical regression model. Hepatol Int 2012;6(4):735-743. https://doi.org/10.1007/s12072-012-9344-9

7. Wu T, Li J, Shao L, Xin J, Jiang L, Zhou Q, et al. Development of diagnostic criteria and a prognostic score for hepatitis B virus-related acute-on-chronic liver failure, (in eng). Gut 2018;67(12):2181-2191. https://doi.org/10.1136/ gutjnl-2017-314641

8. Chen T, Yang Z, Choudhury AK, Al Mahtab M, Li J, Chen Y, et al. Complications constitute a major risk factor for mortality in hepatitis B virus-related acute-on-chronic liver failure patients: a multi-national study from the Asia-Pacific region, (in eng). Hepatol Int 2019;13(6):695-705. https://doi.org/10. 1007/s12072-019-09992-x

9. Yang F, Liu Y, Zeng B, Chu J, Hu H, Yang Y, et al. Noninvasive assessment of liver fibrosis for predicting acute-on-chronic liver failure in patients with chronic hepatitis B. Hepatol Int 2021. https://doi.org/10.1007/s12072-020-10106-1 
10. Jin JY, Zheng YB, Zheng J, Liu J, Mao YJ, Chen SG, et al. 2D shear wave elastography combined with MELD improved prognostic accuracy in patients with acute-on-chronic hepatitis B liver failure, (in eng). Eur Radiol 2018;28(10):4465-4474. https://doi.org/10.1007/s00330-018-5336-Z
Publisher's Note Springer Nature remains neutral with regard to jurisdictional claims in published maps and institutional affiliations. 